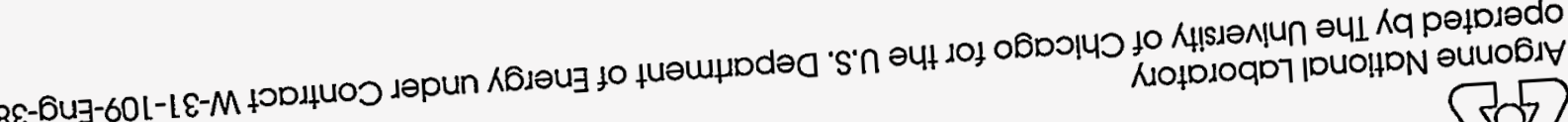

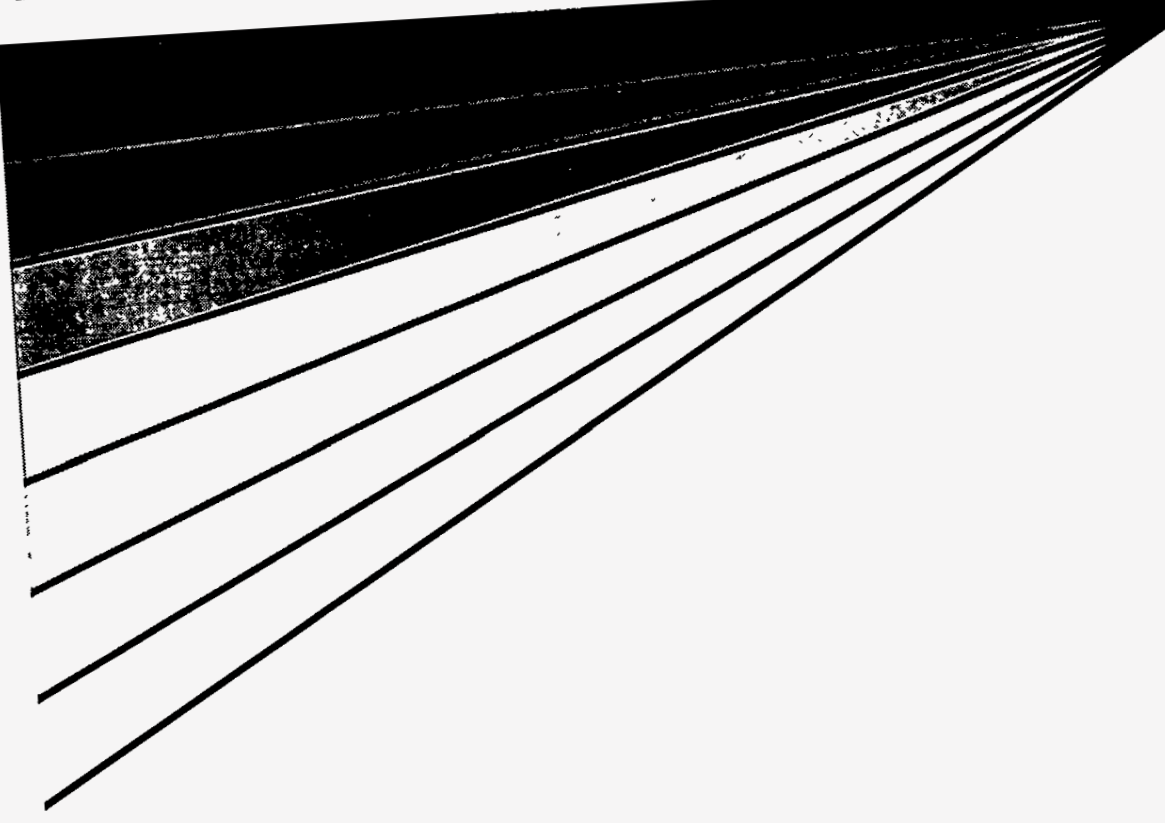

RECEIVED

NOV 041996

OSTI

966 K אin

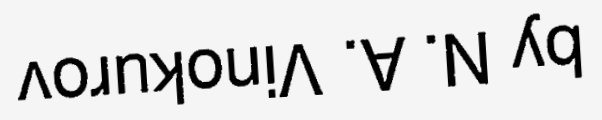


Argonne National Laboratory, with facilities in the states of Illinois and Idaho, is owned by the United States government, and operated by The University of Chicago under the provisions of a contract with the Department of Energy.

\section{DISCLAIMER}

This report was prepared as an account of work sponsored by an agency of the United States Government. Neither the United States Government nor any agency thereof, nor any of their employees, makes any warranty, express or implied, or assumes any legal liability or responsibility for the accuracy, completeness, or usefulness of any information, apparatus, product, or process disclosed, or represents that its use would not infringe privately owned rights. Reference herein to any specific commercial product, process, or service by trade name, trademark, manufacturer, or otherwise, does not necessarily constitute or imply its endorsement, recommendation, or favoring by the United States Government or any agency thereof. The views and opinions of authors expressed herein do not necessarily state or reflect those of the United States Government or any agency thereof.

Reproduced from the best available copy.

Available to DOE and DOE contractors from the Office of Scientific and Technical Information

P.O. Box 62

Oak Ridge, TN 37831

Prices available from (423) 576-8401

Available to the public from the

National Technical Information Service

U.S. Department of Commerce

5285 Port Royal Road

Springfield, VA 22161 
Distribution Category: Atomic, Molecular, and Chemical Physics (UC-411)

ARGONNE NATIONAL LABORATORY

9700 South Cass Avenue

Argonne, Illinois 60439

ANL/APS/TB-27

\title{
The Integral Equation for a High Gain FEL
}

\author{
N. A. Vinokurov
}

Experimental Facilities Division Advanced Photon Source

July 1996

work sponsored by

U.S. DEPARTMENT OF ENERGY

Office of Energy Research 



\section{DISCLAIMER}

This report was prepared as an account of work sponsored by an agency of the United States Government. Neither the United States Government nor any agency thereof, nor any of their employees, makes any warranty, express or implied, or assumes any legal liability or responsibility for the accuracy, completeness, or usefulness of any information, apparatus, product, or process disclosed, or represents that its use would not infringe privately owned rights. Reference herein to any specific commercial product, process, or service by trade name, trademark, manufacturer, or otherwise does not necessarily constitute or imply its endorsement, recommendation, or favoring by the United States Government or any agency thereof. The views and opinions of authors expressed herein do not necessarily state or reflect those of the United States Government or any agency thereof. 


\section{DISCLAIMER}

Portions of this document may be illegible in electronic image products. Images are produced from the best available original document. 


\title{
THE INTEGRAL EQUATION FOR A HIGH GAIN FEL
}

\author{
N. A. Vinokurov *
}

\section{Introduction}

The theory of a high gain free electron laser (FEL) is now well developed (e.g., see [1]). In this paper I derive the equation for the electron distribution function, which is valid for FELs with a longitudinally inhomogeneous magnetic system (which may include, in particular, dispersive sections, quadrupole lenses, and simply empty spaces between the undulator sections), magnetic field errors in undulators, and some other options. The integral form of the equation may be useful for numerical calculations.

\section{Calculation of the Radiation Field}

Consider the electron beam propagating inside a long planar undulator. The Fourier transform $A_{x}$ of the transverse horizontal component of vector potential (Lorentz gauge) is given by [2]

$$
A_{x}(\vec{r})=\frac{1}{c} \int \frac{j_{x}\left(\vec{r}^{\prime}\right) e^{i k\left|\vec{r}-\vec{r}^{\prime}\right|}}{\left|\vec{r}-\vec{r}^{\prime}\right|} d^{3} r^{\prime}+A_{0 x}(\vec{r}),
$$

where $c$ is the light velocity, $k=\omega / c$ is the wave number, $j_{x}$ is the component of a current density, and $A_{0 x}$ describes the external electromagnetic wave. In the paraxial approximation

$$
\left|\vec{r}-\vec{r}^{\prime}\right| \approx z-z^{\prime}+\frac{\left(x-x^{\prime}\right)^{2}+\left(y-y^{\prime}\right)^{2}}{2\left(z-z^{\prime}\right)},
$$

and the electric field is

$$
E=i k A_{x}=\frac{i k}{c} \int_{0}^{z} \iint \frac{j_{x}\left(\vec{r}^{\prime}\right) e^{i k\left(z-z^{\prime}\right)} e^{i k \frac{\left(x-x^{\prime}\right)^{2}+\left(y-y^{\prime}\right)^{2}}{2\left(z-z^{\prime}\right)}}}{z-z^{\prime}} d x^{\prime} d y^{\prime} d z^{\prime}+E_{0} .
$$

The fast particle motion along the $z$ axis (i.e., the varying of $j_{x}\left(\vec{r}^{\prime}\right)$ almost like $e^{i k z^{\prime}}$ ) was taken into account in Eq. (3). If the equation for the equilibrium trajectory in the undulator is

$$
\frac{d x}{d z}=\frac{K(z)}{\gamma} \sin \left[k_{w} z+\varphi(z)\right]
$$

\footnotetext{
* on leave from Budker Institute of Nuclear Physics
} 
where $\gamma$ is the Lorentz factor for the equilibrium particle, $k_{w}$ and $K$ are the wavenumber and the dimensionless vector potential amplitude of the undulator, then

$$
j_{x} \approx j_{z} \frac{K(z)}{\gamma} \sin \left[k_{w} z+\varphi(z)\right]
$$

and

$$
E=\frac{i k}{\gamma c} \iiint \frac{j_{z}\left(\vec{r}^{\prime}\right) K \sin \left(k_{w} z^{\prime}+\varphi\right) e^{i k\left(z-z^{\prime}\right)+i k \frac{\left(x-x^{\prime}\right)^{2}+\left(y-y^{\prime}\right)^{2}}{2\left(z-z^{\prime}\right)}}}{z-z^{\prime}} d x^{\prime} d y^{\prime} d z^{\prime}+E_{0} .
$$

\section{The Vlasov Equation}

To calculate the current density $j_{z}$, we may use the unperturbed (by the radiation field) particle trajectories:

$$
\begin{aligned}
& x(z)=\xi_{1}\left(z, x_{0}, \dot{x}_{0}, y_{0}, \dot{y}_{0}\right)=x_{1}\left(z, x_{0}, \dot{x}_{0}, y_{0}, \dot{y}_{0}\right) \\
& +\int_{0}^{z} \frac{K}{\gamma}\left[1+\frac{\alpha}{2} k_{w}^{2} x_{1}^{2}\left(z^{\prime}, x_{0}, \dot{x}_{0}, y_{0}, \dot{y}_{0}\right)+\frac{1-\alpha}{2} k_{w}^{2} y_{1}^{2}\left(z^{\prime}, x_{0}, \dot{x}_{0}, y_{0}, \dot{y}_{0}\right)\right] \sin \left(k_{w} z^{\prime}+\varphi\right) d z^{\prime} \\
& y(z)=y_{1}\left(z, x_{0}, \dot{x}_{0}, y_{0}, \dot{y}_{0}\right) .
\end{aligned}
$$

Here and below, point notation (i.e., $\dot{x}$ or $\dot{y}$ ) is used for the derivative with respect to $z$, which will be an independent variable (instead of time), and " 0 " indicates the initial conditions (at $z=0$ ). $\alpha=0$ for the planar undulator without sextupole focusing. In the more general case, these trajectories depend also on the initial energy, but for the paraxial motion we may neglect this dependence. As the interaction of particles with light changes essentially only the particle energy, the 6-dimensional distribution function may be written in the form:

$$
\begin{aligned}
& F_{6}(x, \dot{x}, y, \dot{y}, t, \Delta, z)= \\
& \int F\left(\tau, \Delta, z, x_{0}, \dot{x}_{0}, y_{0}, \dot{y}_{0}\right) \delta\left(x-\xi_{1}\right) \delta\left(\dot{x}-\dot{\xi}_{1}\right) \delta\left(y-y_{1}\right) \delta\left(\dot{y}-\dot{y}_{1}\right) d x_{0} d \dot{x}_{0} d y_{0} d \dot{y}_{0},
\end{aligned}
$$

where

$$
\tau=t-\int_{0}^{z}\left[1+\frac{1}{2 \gamma^{2}}+\frac{\dot{\xi}_{1}^{2}\left(z^{\prime}\right)+\dot{y}_{1}^{2}\left(z^{\prime}\right)}{2}\right] \frac{d z^{\prime}}{c}
$$




$$
\int F_{6} d x d \dot{x} d y d \dot{y} d \Delta d t=1
$$

and $(1+\Delta) \gamma$ is the Lorentz factor. For the unperturbed motion at the equilibrium energy, $\tau$ denotes the moment of time when the particle enters the undulator. Therefore $\tau$ is a convenient longitudinal coordinate of a particle. The "mixed" distribution function F obeys the Liouville equation:

$$
\frac{\partial F}{\partial z}+\frac{\partial F}{\partial \tau}\left[\frac{1}{v_{z}}-\frac{1}{c}\left(1+\frac{1}{2 \gamma^{2}}+\frac{\dot{\xi}_{1}^{2}+\dot{y}_{1}^{2}}{2}\right)\right]+\frac{\partial F}{\partial \Delta} \frac{e E_{x}\left(\xi_{1}, y_{1}, z, t\right) \dot{\xi}_{1}}{\gamma m c^{2}}=0,
$$

where $m$ is the electron mass, and $E_{x}$ is the component of the wave electric field. Defining the slow varying amplitude of the electric field as

$$
\begin{aligned}
& E_{x}(x, y, z, t)=A(x, y, z, t) e^{i k_{0}(z-c t)}+c . c . \\
& k_{0} \approx 2 \gamma_{\|}^{2} k_{w} \\
& \gamma_{\|}=\frac{\gamma}{\sqrt{1+K^{2} / 2}}
\end{aligned}
$$

and neglecting all but the slow (by $z$ ) part of longitudinal force, one can obtain

$$
\begin{aligned}
& \frac{\partial F}{\partial z}-\frac{\Delta}{\gamma_{\|}^{2} c} \frac{\partial F}{\partial \tau}= \\
& -\frac{\partial F}{\partial \Delta} \frac{e(J J) K}{\gamma^{2} m c^{2}} \operatorname{Im}\left[A\left(x_{1}, y_{1}, z, \tau+t_{1}\right) e^{\left.i \varphi-i k_{0}\left(\int_{0}^{z} \frac{\dot{x}_{1}^{2}+\dot{y}_{1}^{2}+k_{x}^{2} x_{1}^{2}+k_{y}^{2} y_{1}^{2}}{2} d z^{\prime}+c \tau\right)+i k_{w} z-i k_{0} \int_{0}^{z} \frac{d z^{\prime}}{2 \gamma_{\|}^{2}}\right]},\right.
\end{aligned}
$$

where

$$
(J J)=J_{0}\left(\frac{K^{2}}{4+2 K^{2}}\right)-J_{1}\left(\frac{K^{2}}{4+2 K^{2}}\right)
$$

is the standard combination of the Bessel functions, which describes the reduction of the particlewave interaction due to the longitudinal velocity modulation in the planar undulator, and 


$$
t_{1}=\int_{0}^{z}\left[1+\frac{1}{2 \gamma_{\|}^{2}}+\frac{\dot{x}_{1}^{2}\left(z^{\prime}\right)+\dot{y}_{1}^{2}\left(z^{\prime}\right)+k_{x}^{2} x_{1}^{2}+k_{y}^{2} y_{1}^{2}}{2}\right] \frac{d z^{\prime}}{c}
$$

Wave numbers $k_{x}$ and $k_{y}$ describe focusing by the inhomogeneity of the undulator field:

$$
k_{x}=\sqrt{\alpha} \frac{k_{w} K}{\sqrt{2} \gamma}, \quad k_{y}=\sqrt{1-\alpha} \frac{k_{w} K}{\sqrt{2} \gamma} \text {. }
$$

\section{The Expression of Radiation Field through the Distribution Function}

For an electron bunch having total charge $\mathrm{Q}$, the longitudinal current density is given by

$$
j_{z}=Q \int F_{6} d \dot{x} d \dot{y} d \Delta
$$

or, using Eq. (8),

$$
\begin{aligned}
& j_{z}=Q \int F\left(t-\int_{0}^{2}\left[1+\frac{1}{2 \gamma^{2}}+\frac{\dot{\xi}_{1}^{2}\left(z^{\prime}\right)+\dot{y}_{1}^{2}\left(z^{\prime}\right)}{2}\right] \frac{d z^{\prime}}{c}, \Delta, z, x_{0}, \dot{x}_{0}, y_{0}, \dot{y}_{0}\right) \\
& \times \delta\left(x-\xi_{1}\right) \delta\left(y-y_{1}\right) d x_{0} d \dot{x}_{0} d y_{0} d \dot{y}_{o} .
\end{aligned}
$$

In the stationary case, it is more natural to normalize the average value of $F_{6}$ :

$$
\begin{aligned}
& \left\langle F_{6}\right\rangle=\lim \frac{1}{T} \int_{0}^{T} F_{6} d t, \quad T \rightarrow \infty \\
& \int\left\langle F_{6}\right\rangle d x d \dot{x} d y d \dot{y} d \Delta=1 .
\end{aligned}
$$

Then the longitudinal current density will be

$$
j_{z}=I_{0} \int F_{6} d \dot{x} d \dot{y} d \Delta
$$

or 
$j_{z}=I_{0} \int F\left(t-\int_{0}^{z}\left[1+\frac{1}{2 \gamma^{2}}+\frac{\dot{\xi}_{1}^{2}\left(z^{\prime}\right)+\dot{y}_{1}^{2}\left(z^{\prime}\right)}{2}\right] \frac{d z^{\prime}}{c}, \Delta, z, x_{0}, \dot{x}_{0}, y_{0}, \dot{y}_{0}\right)$

$\times \delta\left(x-\xi_{1}\right) \delta\left(y-y_{1}\right) d x_{0} d \dot{x}_{0} d y_{0} d \dot{y}_{o} d \Delta$

where $I_{0}$ is the average beam current.

The expression for the field amplitude $A$, defined in Eq. (12), is coming from Eq. (6):

$A=A_{0}$

$+\frac{i k_{0} e^{-i k_{0}(z-c t)}}{\gamma_{c}} \iiint_{0}^{z} \frac{j_{z}\left(\vec{r}^{\prime}, t-\frac{z-z^{\prime}}{c}-\frac{\left(x-x^{\prime}\right)^{2}+\left(y-y^{\prime}\right)^{2}}{2 c\left(z-z^{\prime}\right)}\right) K \sin \left(k_{w} z^{\prime}+\varphi\right)}{z-z^{\prime}} d x^{\prime} d y^{\prime} d z^{\prime}$.

Substituting Eq. (20') into Eq. (22) gives:

$$
\begin{aligned}
& A=\frac{i k_{0} I_{0} e^{-i k_{0}(z-c t)}}{\gamma c} \int_{0}^{z} F\left(t-\frac{z}{c}-\frac{\left(x-\xi_{1}\left(z^{\prime}\right)\right)^{2}+\left(y-y_{1}\left(z^{\prime}\right)\right)^{2}}{2 c\left(z-z^{\prime}\right)}\right. \\
& \left.-\int_{0}^{z^{\prime}}\left[\frac{1}{2 \gamma^{2}}+\frac{\dot{\xi}_{1}^{2}\left(z^{\prime \prime}\right)+\dot{y}_{1}^{2}\left(z^{\prime \prime}\right)}{2}\right] \frac{d z^{\prime \prime}}{c}, \Delta, z, x_{0}, \dot{x}_{0}, y_{0}, \dot{y}_{0}\right) \\
& \times \frac{K \sin \left(k_{w} z^{\prime}+\varphi\right)}{z-z^{\prime}} d x_{0} d \dot{x}_{0} d y_{0} d \dot{y}_{o} d \Delta d z^{\prime}+A_{0} .
\end{aligned}
$$

Assuming, that only the slow varying component of the function $F e^{i \omega_{o} \tau}$ makes a sufficient contribution to the integral in Eq. (23), we may neglect some rapidly oscillating terms:

$$
\begin{aligned}
& \sin \left(k_{w} z^{\prime}+\varphi\right) e^{-i \omega_{0} \tau} e^{i \omega_{0} \tau} F\left(t-\frac{z}{c}-\frac{\left(x-\xi_{1}\left(z^{\prime}\right)\right)^{2}+\left(y-y_{1}\left(z^{\prime}\right)\right)^{2}}{2 c\left(z-z^{\prime}\right)}\right. \\
& \left.-\int_{0}^{z^{\prime}}\left[\frac{1}{2 \gamma^{2}}+\frac{\dot{\xi}_{1}^{2}\left(z^{\prime \prime}\right)+\dot{y}_{1}^{2}\left(z^{\prime \prime}\right)}{2}\right] \frac{d z^{\prime \prime}}{c}, \Delta, z, x_{0}, \dot{x}_{0}, y_{0}, \dot{y}_{0}\right) \approx
\end{aligned}
$$

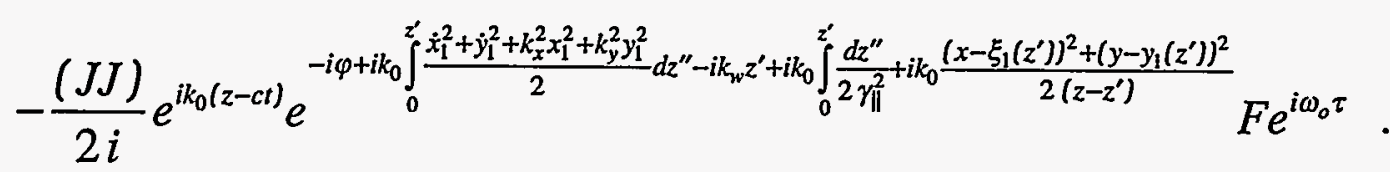


Actually, we simply change $\sin \left(k_{w} z^{\prime}+\varphi\right)$ to $i e^{-i\left(k_{w} z^{\prime}+\varphi\right)}(J J) / 2$. Thus, the expression in Eq. (23) is changed to

$$
\begin{aligned}
& A=-\frac{k_{0} I_{0} e^{-i k_{0}(z-c t)}}{2 \gamma c} \int_{0}^{z} \int F\left(t-\frac{z}{c}-\frac{\left(x-x_{1}\left(z^{\prime}\right)\right)^{2}+\left(y-y_{1}\left(z^{\prime}\right)\right)^{2}}{2 c\left(z-z^{\prime}\right)}\right. \\
& \left.-\int_{0}^{z^{\prime}}\left[\frac{1}{2 \gamma_{\|}^{2}}+\frac{\dot{x}_{1}^{2}+\dot{y}_{1}^{2}+k_{x}^{2} x_{1}^{2}+k_{y}^{2} y_{1}^{2}}{2}\right] \frac{d z^{\prime \prime}}{c}, \Delta, z, x_{0}, \dot{x}_{0}, y_{0}, \dot{y}_{0}\right) \\
& \times \frac{K(J J)}{z-z^{\prime}} e^{-i \varphi-i k_{w} z^{\prime}} d x_{0} d \dot{x}_{0} d y_{0} d \dot{y}_{0} d \Delta d z^{\prime}+A_{0} .
\end{aligned}
$$

\section{The Final Equation}

Substituting Eq. (25) into Eq. (15), one can easily obtain the final equation:

$$
\begin{aligned}
& \frac{\partial F}{\partial z}-\frac{\Delta}{\gamma_{\|}^{2} c} \frac{\partial F}{\partial \tau}= \\
& \frac{\partial F}{\partial \Delta} \operatorname{Im}\left\{\frac{e(J J)_{0} K_{0}}{\gamma^{2} m c^{2}} B A_{0}\left(x_{1}, y_{1}, z, \tau+t_{1}\right) e^{i k_{0}\left(z-c \tau-c t_{1}\right)} e^{i k_{w} z}\right. \\
& +\frac{k_{w} D^{2} B}{4} \int_{0}^{z} \frac{\bar{B} e^{i k_{w}\left(z-z^{\prime}\right)}}{z-z^{\prime}} \int F\left(\tau+\int_{z^{\prime}}^{z} \frac{d z^{\prime \prime}}{2 c \gamma_{\|}^{2}}-\frac{\left[x_{1}(z)-x_{1}^{\prime}\left(z^{\prime}\right)\right]^{2}+\left[y_{1}(z)-y_{1}^{\prime}\left(z^{\prime}\right)\right]^{2}}{2 c\left(z-z^{\prime}\right)}\right. \\
& +\int_{0}^{z} \frac{\dot{x}_{1}^{2}+\dot{y}_{1}^{2}+k_{x}^{2} x_{1}^{2}+k_{y}^{2} y_{1}^{2}}{2} \frac{d z^{\prime \prime}}{c}-\int_{0}^{z^{\prime}} \frac{\dot{x}_{1}^{\prime 2}+\dot{y}_{1}^{\prime 2}+k_{x}^{2} x_{1}^{\prime 2}+k_{y}^{2} y_{1}^{\prime 2}}{2} \frac{d z^{\prime \prime}}{c}, \\
& \left.\left.\Delta^{\prime}, z^{\prime}, x_{0}^{\prime}, \dot{x}_{0}^{\prime}, y_{0}^{\prime}, \dot{y}_{0}^{\prime}\right) d x_{0}^{\prime} d \dot{x}_{0}^{\prime} d y_{0}^{\prime} d \dot{y}_{o}^{\prime} d \Delta^{\prime} d z^{\prime}\right\}
\end{aligned}
$$

where $K_{0}$ and $(J J)_{0}$ are constant,

$$
\begin{aligned}
& 1+\frac{K_{0}^{2}}{2}=\frac{2 \gamma^{2} k_{w}}{k_{0}}, \\
& B=\frac{K(J J)}{K_{0}(J J)_{0}} e^{i \varphi},
\end{aligned}
$$

and 


$$
D=4 \sqrt{\frac{e I_{0}}{\gamma m c^{3}}} \sqrt{\frac{K_{0}^{2}}{4+2 K_{0}^{2}}}(J J)_{0} .
$$

Equation (26) may be written as an integral equation:

$$
\begin{aligned}
& F\left(\tau, \Delta, z, x_{0}, \dot{x}_{0}, y_{0}, \dot{y}_{0}\right)= \\
& F\left(\tau+\frac{\Delta}{c} \int_{0}^{z} \frac{d z^{\prime}}{\gamma_{\|}^{2}}, \Delta, 0, x_{0}, \dot{x}_{0}, y_{0}, \dot{y}_{0}\right)+\int_{0}^{z} R\left(\tau+\frac{\Delta}{c} \int_{z^{\prime}}^{z} \frac{d z^{\prime \prime}}{\gamma_{\|}^{2}}, \Delta, z^{\prime}\right) d z^{\prime},
\end{aligned}
$$

where $R(\tau, \Delta, z)$ is the right-hand side of Eq. (25). The physical interpretation of Eqs. (26) and (30) is simple. We consider the electron beam as a set of thin beams, each having its own initial conditions $x_{0}, \dot{x}_{0}, y_{0}, \dot{y}_{0}$ and corresponding trajectory $x_{1}\left(z, x_{0}, \dot{x}_{0}, y_{0}, \dot{y}_{0}\right)$,

$y_{1}\left(z, x_{0}, \dot{x}_{0}, y_{0}, \dot{y}_{0}\right)$. Electrons moving along the undulator do not change their trajectories (this is an approximation). Therefore we have, in fact, one-dimensional motion along each trajectory (see the left side of Eq. (26)). The right side of Eq. (26) contains the longitudinal force on the electron, moving along the above-mentioned trajectory. The force is the sum of the contributions of the other electrons, moving along other trajectories $x_{1}\left(z, x_{0}^{\prime}, \dot{x}_{0}^{\prime}, y_{0}^{\prime}, \dot{y}_{0}^{\prime}\right)$,

$$
y_{1}\left(z, x_{0}^{\prime}, \dot{x}_{0}^{\prime}, y_{0}^{\prime}, \dot{y}_{0}^{\prime}\right) \text {. }
$$

\section{The Case of a Small Signal}

It is enough to put $\partial F_{0} / \partial \Delta$ instead of $\partial F / \partial \Delta$ into right side of Eq. (26) to obtain the linear equation for a small signal. We assume that $F_{0}$ is the "smooth" part of the distribution function, which does not make a contribution to the radiation field, and keep notation $F$ for the small variable component of the distribution function. Defining the current of the stream

$$
J=\int F d \Delta
$$

one can obtain the linear equation for it from Eq. (30): 


$$
\begin{aligned}
& J\left(\tau, z, x_{0}, \dot{x}_{0}, y_{0}, \dot{y}_{0}\right)=\int F\left(\tau+\frac{\Delta}{c} \int_{0}^{z} \frac{d z^{\prime}}{\gamma_{\|}^{2}}, \Delta, 0, x_{0}, \dot{x}_{0}, y_{0}, \dot{y}_{0}\right) d \Delta \\
& +\int_{0}^{z} \int \frac{\partial F_{0}}{\partial \Delta} \operatorname{Im}\left\{\frac{e(J J)_{0} K_{0}}{\gamma^{2} m c^{2}} B A_{0}\left(x_{1}\left(z^{\prime}\right), y_{1}\left(z^{\prime}\right), z^{\prime}, \tau+\frac{\Delta}{c} \int_{z^{\prime}}^{z} \frac{d z^{\prime \prime}}{\gamma_{\|}^{2}}+t_{1}\left(z^{\prime}\right)\right)\right. \\
& \left.\times e^{i k_{0}\left[z^{\prime}-c \tau-\Delta \int_{z^{2}}^{2} \frac{d z^{\prime \prime}}{\gamma_{\|}^{2}}-c t_{1}\left(z^{\prime}\right)\right]} e^{i k_{w} z^{\prime}}\right\} d \Delta d z^{\prime} \\
& +\frac{k_{w} D^{2}}{4} \int_{0}^{z} \int_{0}^{z} \frac{\partial F_{0}}{\partial \Delta} \operatorname{Im}\left\{\int_{0}^{z^{\prime}} \frac{\bar{B} e^{i k_{w}\left(z^{\prime}-z^{\prime \prime}\right)}}{z^{\prime}-z^{\prime \prime}}\right. \\
& \times \int_{0} J\left(\tau+\frac{\Delta}{c} \int_{z^{\prime}}^{z} \frac{d z^{\prime \prime}}{\gamma_{\|}^{2}}+\int_{z^{\prime \prime}}^{z^{\prime}} \frac{d z^{\prime \prime \prime}}{2 c \gamma_{\|}^{2}}-\frac{\left[x_{1}\left(z^{\prime}\right)-x_{1}^{\prime}\left(z^{\prime \prime}\right)\right]^{2}+\left[y_{1}\left(z^{\prime}\right)-y_{1}^{\prime}\left(z^{\prime \prime}\right)\right]^{2}}{2 c\left(z^{\prime}-z^{\prime \prime}\right)}\right. \\
& \left.+\int_{0}^{z^{\prime}} \frac{\dot{x}_{1}^{2}+\dot{y}_{1}^{2}+k_{x}^{2} x_{1}^{2}+k_{y}^{2} y_{1}^{2}}{2} \frac{d z^{\prime \prime \prime}}{c}-\int_{0}^{z^{\prime \prime}} \frac{\dot{x}_{1}^{\prime 2}+\dot{y}_{1}^{\prime 2}+k_{x}^{2} x_{1}^{\prime 2}+k_{y}^{2} y_{1}^{\prime 2}}{2} \frac{d z^{\prime \prime \prime}}{c}, z^{\prime \prime}, x_{0}^{\prime}, \dot{x}_{0}^{\prime}, y_{0}^{\prime}, \dot{y}_{0}^{\prime}\right) \\
& \left.\times d x_{0}^{\prime} d \dot{x}_{0}^{\prime} d y_{0}^{\prime} d \dot{y}_{0}^{\prime} d z^{\prime \prime}\right\} d \Delta d z^{\prime} .
\end{aligned}
$$

The slow variable may be defined as follows:

$$
\begin{aligned}
& F=f e^{-i \omega_{0} \tau}+c . c . \\
& J=j e^{-i \omega_{0} \tau}+c . c .
\end{aligned}
$$


Then Eq. (32) gives:

$$
\begin{aligned}
& j\left(\tau, z, x_{0}, \dot{x}_{0}, y_{0}, \dot{y}_{0}\right)=\int_{-\infty}^{\infty} f\left(\tau, \Delta, 0, x_{0}, \dot{x}_{0}, y_{0}, \dot{y}_{0}\right) e^{-i k_{0} \Delta \frac{\tau}{0} \gamma_{0}^{2}} d \Delta+\frac{e(J J)_{0} K_{0}}{2 i \gamma^{2} m c^{2}} \\
& \times \int_{0}^{z} \Phi\left(k_{0} \int_{z^{\prime}}^{z} \frac{d z^{\prime \prime}}{\gamma_{\|}^{2}}, \tau, z^{\prime}, x_{0}, \dot{x}_{0}, y_{0}, \dot{y}_{0}\right) B\left(z^{\prime}\right) A_{0}\left(x_{1}\left(z^{\prime}\right), y_{1}\left(z^{\prime}\right), z^{\prime}, \tau+\frac{z^{\prime}}{c}+\int_{0}^{z^{\prime}} \frac{d z^{\prime \prime}}{2 c \gamma_{\|}^{2}}\right) \\
& \times e^{-i k_{0} \int_{0}^{z^{\prime} \dot{x}_{1}^{2}+\dot{y}_{1}^{2}+k_{x}^{2} x_{1}^{2}+k_{y}^{2} y_{1}^{2}}} \frac{z^{\prime \prime}}{2} e^{i k_{w} z^{\prime}-i k_{o} \int \frac{z^{\prime}}{02 z^{\prime \prime}}} d z^{\prime} \\
& +\frac{k_{w} D^{2}}{8 i} \int_{0}^{z} \Phi\left(k_{0} \int_{z^{\prime}}^{z} \frac{d z^{\prime \prime}}{\gamma_{\|}^{2}}, \tau, z^{\prime}, x_{0}, \dot{x}_{0}, y_{0}, \dot{y}_{0}\right) B\left(z^{\prime}\right) \int_{0}^{z^{\prime}} \frac{\overline{B\left(z^{\prime \prime}\right)}}{z^{\prime}-z^{\prime \prime}} e^{i k_{w}\left(z^{\prime}-z^{\prime \prime}\right)-i k_{o}} \int_{z^{\prime}-2 \gamma_{\|}^{\prime}}^{z^{\prime \prime}}
\end{aligned}
$$

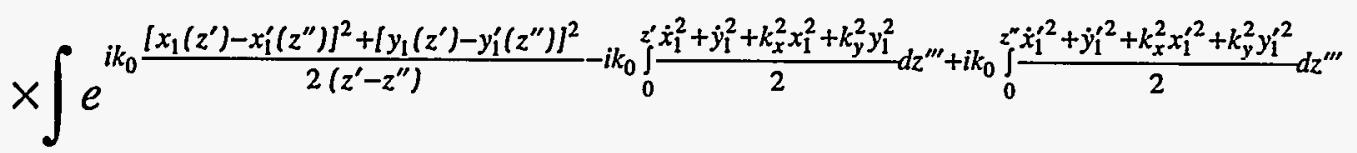

$$
\begin{aligned}
& \times j\left(\tau+\int_{z^{\prime \prime}}^{z^{\prime}} \frac{d z^{\prime \prime \prime}}{2 c \gamma_{\|}^{2}}, z^{\prime \prime}, x_{0}^{\prime}, \dot{x}_{0}^{\prime}, y_{0}^{\prime}, \dot{y}_{0}^{\prime}\right) d x_{0}^{\prime} d \dot{x}_{0}^{\prime} d y_{0}^{\prime} d \dot{y}_{0}^{\prime} d z^{\prime \prime} d z^{\prime}
\end{aligned}
$$

where

$$
\Phi(\kappa)=\int_{-\infty}^{\infty} \frac{\partial F_{0}}{\partial \Delta} e^{-i \kappa \Delta} d \Delta .
$$

If, for example,

$$
F_{0}=\frac{1}{\sqrt{2 \pi} \sigma} e^{-\frac{(\Delta-\varepsilon)^{2}}{2 \sigma^{2}}} f_{0}
$$

where $\varepsilon, \sigma$, and $f_{0}$ are functions of $z, \tau, x_{0}, \dot{x}_{0}, y_{0}, \dot{y}_{0}$, then

$$
\Phi(\kappa)=i \kappa e^{-i \kappa \varepsilon-\frac{\kappa^{2} \sigma^{2}}{2}} f_{0} .
$$


If $F_{0}$ does not depend on $\tau$ and $z$, the Fourier transformation of Eq. (35) gives:

$$
\begin{aligned}
& j_{\omega}\left(z, x_{0}, \dot{x}_{0}, y_{0}, \dot{y}_{0}\right)=\int_{-\infty}^{\infty} f_{\omega}\left(\Delta, 0, x_{0}, \dot{x}_{0}, y_{0}, \dot{y}_{0}\right) e^{-i k_{0} \Delta \int_{0}^{2} \frac{d z^{\prime}}{\gamma_{\|}^{2}}} d \Delta+\frac{e(J J)_{0} K_{0}}{2 i \gamma^{2} m c^{2}} \\
& \times \int_{0}^{z} \Phi\left(k_{0} \int_{z^{\prime}}^{z} \frac{d z^{\prime \prime}}{\gamma_{\|}^{2}}, x_{0}, \dot{x}_{0}, y_{0}, \dot{y}_{0}\right) B\left(z^{\prime}\right) A_{0 \omega}\left(x_{1}\left(z^{\prime}\right), y_{1}\left(z^{\prime}\right), z^{\prime}\right) e^{-i \frac{\omega}{c}\left(z^{\prime}+z_{0}^{\prime} \frac{d z^{\prime \prime}}{2 \gamma_{\|}^{2}}\right)} \\
& \times e^{-i k_{0} \int_{0}^{z^{\prime} \dot{x}_{1}^{2}+\dot{y}_{1}^{2}+k_{x}^{2} x_{1}^{2}+k_{y}^{2} y_{1}^{2}}} \frac{z^{\prime \prime}}{2} e^{i k_{w} z^{\prime}-i k_{o} \int_{0}^{z^{\prime}} \frac{d z^{\prime \prime}}{2 \gamma_{\|}^{2}}} d z^{\prime} \\
& +\int_{0}^{z} \int \mathrm{K}\left(z, z^{\prime}, x_{0}, \dot{x}_{0}, y_{0}, \dot{y}_{0}, x_{0}^{\prime}, \dot{x}_{0}^{\prime}, y_{0}^{\prime}, \dot{y}_{0}^{\prime}\right) j_{\omega}\left(z^{\prime}, x_{0}^{\prime}, \dot{x}_{0}^{\prime}, y_{0}^{\prime}, \dot{y}_{0}^{\prime}\right) d x_{0}^{\prime} d \dot{x}_{0}^{\prime} d y_{0}^{\prime} d \dot{y}_{o}^{\prime} d z^{\prime} \text {, }
\end{aligned}
$$

where

$$
\begin{aligned}
& \mathrm{K}\left(z, z^{\prime}, x_{0}, \dot{x}_{0}, y_{0}, \dot{y}_{0}, x_{0}^{\prime}, \dot{x}_{0}^{\prime}, y_{0}^{\prime}, \dot{y}_{0}^{\prime}\right)= \\
& \frac{k_{w} D^{2}}{8 i} \int_{z^{\prime}}^{z} \Phi\left(k_{0} \int_{z^{\prime \prime}}^{z} \frac{d z^{\prime \prime \prime}}{\gamma_{\|}^{2}}, x_{0}, \dot{x}_{0}, y_{0}, \dot{y}_{0}\right) \frac{B\left(z^{\prime \prime}\right) \overline{B\left(z^{\prime}\right)}}{z^{\prime \prime}-z^{\prime}} e^{i k_{w}\left(z^{\prime \prime}-z^{\prime}\right)-i\left(k_{o}+\frac{\omega}{c}\right) z_{z^{\prime \prime}}^{z^{\prime \prime}} \frac{d^{\prime \prime \prime}}{2 \gamma_{\|}^{2}}} \\
& \times e^{i k_{0} \frac{\left[x_{1}\left(z^{\prime \prime}\right)-x_{1}^{\prime}\left(z^{\prime}\right)\right]^{2}+\left[y_{1}\left(z^{\prime \prime}\right)-y_{1}^{\prime}\left(z^{\prime}\right)\right]^{2}}{2\left(z^{\prime \prime}-z^{\prime}\right)}-i k_{0}^{z^{\prime \prime}} \int_{0}^{2} \frac{\dot{x}_{1}^{2}+\dot{y}_{1}^{2}+k_{x}^{2} x_{1}^{2}+k_{y}^{2} y_{1}^{2}}{2} d z^{\prime \prime \prime}+i k_{0} \int_{0}^{z_{1}^{\prime} \dot{x}_{1}^{2}+\dot{y}_{1}^{2}+k_{x}^{2} x_{1}^{\prime 2}+k_{y}^{2} y_{1}^{\prime 2}} d z^{\prime \prime \prime}} d z^{\prime \prime} \text {. }
\end{aligned}
$$

\section{References}

[1] K.-J. Kim and M. Xie, Nucl. Instr. and Meth. A331, 359 (1993).

[2] L. D. Landau and E. M. Lifshitz, Classical Theory of Fields, Reading, Massachusetts: Addison-Wesley, 1951. 